\title{
Optical interference effects on the Casimir-Lifshitz force in multilayer structures
}

\author{
Victoria Esteso $\odot,{ }^{1}$ Sol Carretero-Palacios $\odot,{ }^{1,2, *}$ and Hernán Míguez $\oplus^{1, \dagger}$ \\ ${ }^{1}$ Multifunctional Optical Materials Group, Instituto de Ciencia de Materiales de Sevilla (Consejo Superior de Investigaciones \\ Científicas-Universidad de Sevilla), Calle Américo Vespucio 49, 41092 Sevilla, Spain \\ ${ }^{2}$ Departamento de Física de Materiales, Universidad Autónoma de Madrid, 28049 Madrid, Spain
}

(Received 17 June 2019; revised manuscript received 4 February 2020; accepted 4 February 2020; published 12 March 2020)

\begin{abstract}
The Casimir-Lifshitz force $F_{(C-L)}$ between planar objects when one of them is stratified at the nanoscale is herein investigated. Layering results in optical interference effects that give rise to a modification of the optical losses, which, as stated by the fluctuation-dissipation theorem, should affect the Casimir-Lifshitz interaction. On these grounds, we demonstrate that, by nanostructuring the same volume of dielectric materials in diverse multilayer configurations, it is possible to access $F_{(C-L)}$ of attractive or repulsive nature, even getting canceled, at specific separation distances.
\end{abstract}

DOI: 10.1103/PhysRevA.101.033815

\section{INTRODUCTION}

The well-known van der Waals [1] and Casimir-Lifshitz forces (per unit area) [2,3] between polarizable objects, $F_{(C-L)}$, are at the heart of many fluctuation-induced interactions in natural science and, currently, these are the object of study in a variety of technological applications at the nanoscale, including micro- and nanoelectromechanical devices (MEMS and NEMS) [4-7]. On this point, different ways to modify not only the intensity and nature (attractive or repulsive) of $F_{(C-L)}$, but also the Casimir torque, have been proposed [8-14]. Of special importance, due to their versatility for tuning $F_{(C-L)}$, are the strategies in which the optical properties of the interacting objects are modified [15-20]. According to the fluctuation-dissipation theorem [21], the correlation of quantum fluctuations explicitly depends on the dissipation of the interacting materials or, in other words, the Casimir-Lifshitz force between two separated bodies depends on the absorption properties of the materials involved.

In this regard, the use of multilayer structures has been previously proposed to tune $F_{(C-L)}$ and, particularly, periodic multilayers have been suggested due to their high reflectivity properties arising because of their tunable photonic band gap [22-26]. However, while interest has been focused on the presentation of various formulas for calculating $F_{(C-L)}$ in different types of multilayers [27-35], interference effects of electromagnetic (EM) field at each interface in the multilayer structure have been overlooked, since going to imaginary wave numbers in these formulations implies losing track of interference contributions, and their role cannot be identifiable anymore.

Here, we identify a physical mechanism enabling the manipulation of Casimir-Lifshitz force in multilayer dielectric nanostructures due to optical interference effects. Based on

\footnotetext{
*sol.carretero@uam.es

†h.miguez@csic.es
}

the fluctuation-dissipation theorem, which correlates quantum fluctuations and the dissipation properties of the interacting materials, we find that strong absorption modifications within the multilayer structure due to light trapping inside the system at $\mathrm{UV}$ frequencies are responsible for $F_{(C-L)}$ variations. The latter is possible due to the materials combination selected, as they present forces of similar intensity and opposite nature when considered isolated in single slabs approached to the same substrate $[18,36]$. Also, the thin slab thicknesses considered allow strong spatial and spectral absorption modifications within the stratified planar system having an impact on $F_{(C-L)}$.

In order to analyze the potential effect of optical interference on $F_{(C-L)}$, we devise a multilayer structure comprising alternate bilayers $\left(N_{b}\right)$ of silicon dioxide $\left(\mathrm{SiO}_{2}\right)$ and polystyrene (PS) slabs of equal thickness, $d$, parallel to a silicon $(\mathrm{Si})$ wall at a certain separation distance, $d_{0}$. The whole system is immersed in glycerol [see schematic in Fig. 1(a)]. Such choice is based on a previous screening of materials and liquid compounds to find combinations that can provide either repulsive $\left(\mathrm{SiO}_{2}\right.$ - glycerol- $\left.\mathrm{Si}\right)$ or attractive (PS-glycerol$\mathrm{Si}$ ) forces between two semi-infinite materials with a liquid in between.

In the multilayer scheme in Fig. 1(a), each single layer will be characterized by the corresponding dielectric function at $\omega$ frequencies, $\varepsilon_{L}(\omega)=\varepsilon_{L}^{\prime}(\omega)+i \varepsilon_{L}^{\prime \prime}(\omega)$, with $L=$ $-1,0,1, \ldots, 2 N_{b}$, designating the layer position within the arrangement. Within this notation, the infinitely thick Si substrate takes the value $L=-1$, and the glycerol layer between the multilayer and the $\mathrm{Si}$ wall is set to be $L=0$. Thus the first $\mathrm{SiO}_{2}$ layer of the multilayer corresponds to $L=1$, the second one to PS, with $L=2$, and so on.

\section{THEORETICAL FORMALISM}

To calculate $F_{(C-L)}$ between plane-parallel structures we make use of the Lifshitz's theoretical formalism expressed for an arbitrary number of layers in which the EM field and the 
(a)
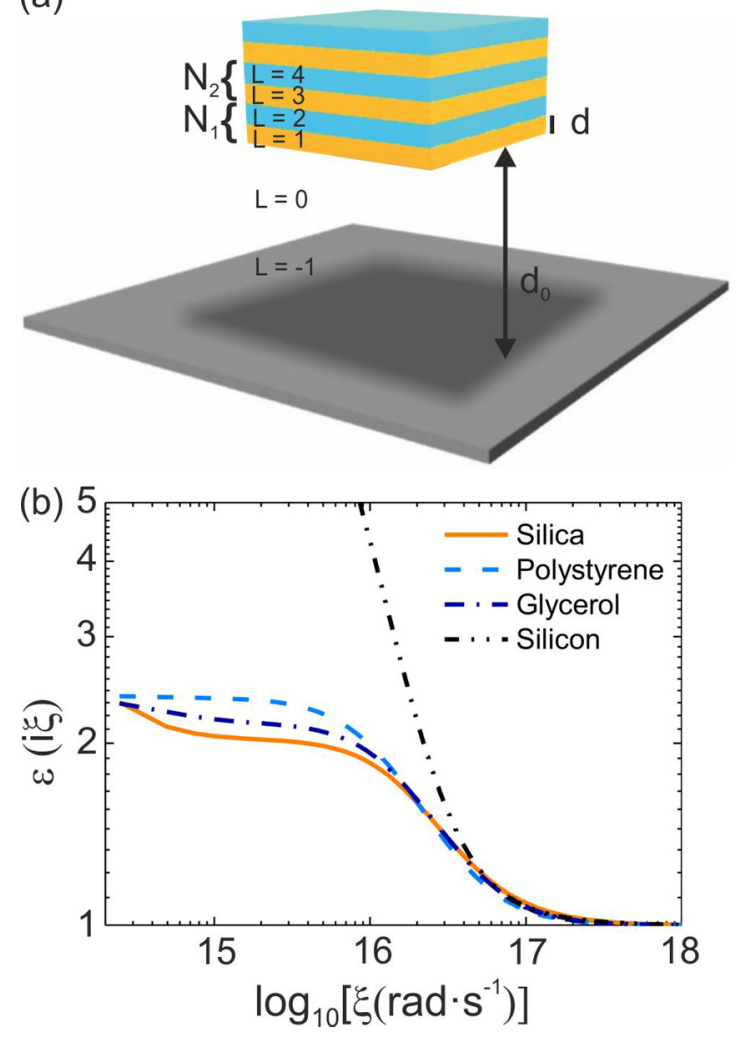

FIG. 1. (a) Schematics of the system under study: a multilayer structure comprising alternate layers of thickness $d$ made of $\mathrm{SiO}_{2}$ and PS materials, immersed in glycerol and facing a $\mathrm{Si}$ wall at a separation distance $d_{0}$. Layers are labeled as $L=-1,0,1, \ldots, 2 N_{b}$. $L=-1$ corresponds to $\mathrm{Si}$ wall, $L=0$ to glycerol, $L=1$ to $\mathrm{SiO}_{2}$, $L=2$ to PS, etc., and $N_{b}$ denotes the total number of $\mathrm{SiO}_{2}-\mathrm{PS}$ bilayers. (b) Dielectric function at Matsubara frequencies, $\varepsilon\left(i \xi_{n}\right)$, for $\mathrm{SiO}_{2}$ (solid line), PS (dashed line), glycerol (dashed dotted line), and $\mathrm{Si}$ (dashed double dotted line), with a doping level $1.1 \times 10^{15} \mathrm{~cm}^{3}$ and resistivity $0.077(\Omega \mathrm{cm})^{-1}$.

material bodies are treated macroscopically [1,3]:

$$
\begin{aligned}
F_{C-L}\left(d_{0}, T\right)= & -\frac{k_{B} T}{\pi} \sum_{n=0}^{\infty}, \int_{0}^{\infty} k_{n}^{(0)} \boldsymbol{k}_{\perp} d \boldsymbol{k}_{\perp} \\
& \times \sum_{j=T E, T M}\left[\frac{e^{2 k_{n}^{(0)} d_{0}}}{R_{j}^{(+)} \cdot R_{j}^{(-)}}-1\right]^{-1} .
\end{aligned}
$$

In the above expression, the wave vector inside glycerol is defined as $\mathbf{K}=\left(\mathbf{k}_{\perp}, k_{n}^{(0)}\right)$, where $\mathbf{k}_{\perp}$ is the wave number in the plane of the interfaces, and $n=0,1,2, \ldots$ enumerates the discrete and infinite Matsubara frequencies $\xi_{n}=\frac{2 \pi k_{B} T}{\hbar} n$, with $k_{B}$ and $\hbar$ being the universal constants and $T$ the temperature. The prime in the summation indicates a factor of $1 / 2$ for $n=0$. Also, $R_{j}^{(+)}\left(n, \boldsymbol{k}_{\perp}\right)=\frac{r_{j}^{(0,+1)}+\Gamma_{L} e^{-2 k_{n}^{(+1)} d_{L}}}{1+r_{j}^{(0,+1)} \Gamma_{L} e^{-2 k_{n}^{(+1)} d_{L}}}$, with $L=1$ and $R_{j}^{(-)}\left(n, \boldsymbol{k}_{\perp}\right)=r_{j}^{(0,-1)}\left(n, \boldsymbol{k}_{\perp}\right)$ are the multiple Fresnel coefficients of the top and bottom surfaces of the glycerol layer, respectively, for transverse electric $(j=\mathrm{TE})$ and transverse magnetic $(j=\mathrm{TM})$ polarizations. These coefficients depend, in turn, upon the simple Fresnel coefficients at Matsubara frequencies:

$$
r_{j}^{(L, L \pm 1)}\left(n, \boldsymbol{k}_{\perp}\right)=\frac{\sigma_{n, j}^{(L \pm 1)} k_{n}^{(L)}-\sigma_{n, j}^{(L)} k_{n}^{(L \pm 1)}}{\sigma_{n, j}^{(L \pm 1)} k_{n}^{(L)}+\sigma_{n, j}^{(L)} k_{n}^{(L \pm 1)}}
$$

with $\sigma_{n, T E}=\mu_{n}, \sigma_{n, T M}=\varepsilon_{n}, \Gamma_{L}^{\left(n, \boldsymbol{k}_{\perp}\right)}=\frac{r_{j}^{(L, L+1)}+\Gamma_{(L+1)} e^{-2 k_{n}^{(L)} d_{L}}}{1+r_{j}^{(L, L+1)} \Gamma_{(L+1)} e^{-2 k_{n}^{(L)} d_{L}}}$, with $d_{L}=d$ for the particular case herein analyzed, and $k_{n}^{(L)}=$ $\left[\boldsymbol{k}_{\perp}^{2}+\varepsilon_{n}^{(L)} \frac{\xi_{n}^{2}}{c^{2}}\right]^{1 / 2}$.

For our nonmagnetic materials, $\mu_{n}=1$. Finally, the permittivity evaluated at Matsubara frequencies is obtained through

$$
\varepsilon_{n}^{(L)} \equiv \varepsilon^{(L)}\left(i \xi_{n}\right)=1+\frac{2}{\pi} \int_{0}^{\infty} \frac{\omega \varepsilon_{L}^{\prime \prime}(\omega)}{\omega^{2}+\xi_{n}^{2}} d \omega .
$$

To evaluate $F_{(C-L)}$, Eq. (3) is applied to the dielectric functions of $\mathrm{SiO}_{2}, \mathrm{PS}$, and $\mathrm{Si}$ tabulated in Refs. [37,38], Refs. [39,40], and Refs. [41,42], accordingly, whereas for glycerol, oscillator models extracted from Ref. [43] are used. Figure 1(b) shows $\varepsilon\left(i \xi_{n}\right)$ as a function of $\xi_{n}$ for the materials here considered. The relation amongst those curves are at the heart of the opposite force nature attained for single slabs made out of $\mathrm{SiO}_{2}$ or PS immersed in glycerol [14,18,36].

Optical properties of the multilayer structure are calculated through the recurrent and iterative expressions provided by the transfer matrix method [44,45] (TMM), a general formalism that allows for computing all optical properties of onedimensional multilayered structures, assuming that the illumination source is placed at a nonabsorbing glycerol medium, whose dielectric function is taken from Refs. [46,47].

\section{RESULTS AND DISCUSSION}

In general, optical properties in periodic multilayer structures show a blueshift of the high reflectance band as the slab thickness of the constitutive materials is reduced, accompanied by a reflectance intensity raise with increasing number of slabs, due to optical interference effects inside the stratified structure. This implies variations of the power absorbed $\left(P_{\mathrm{abs}}\right)$ by the whole system correlated to spectrally and spatially selective enhancements of the electric-field amplitude $[E(\omega, \overline{\boldsymbol{r}})]$, as it follows from

$$
P_{\mathrm{abs}}(\omega)=\frac{1}{2} \int_{0}^{V} \omega \varepsilon_{0} \varepsilon_{L}^{\prime \prime}(\omega, \overline{\boldsymbol{r}})|E(\omega, \overline{\boldsymbol{r}})|^{2} d V,
$$

with $V$ the volume of the system. According to the fluctuationdissipation theorem [21], the current density fluctuations inside a homogeneous material, stemmed from the EM field fluctuations, are proportional to the dissipation of the material, i.e., $\left\langle J_{\alpha}(\omega, \overline{\boldsymbol{r}}) J_{\beta}^{*}\left(\omega^{\prime}, \overline{\boldsymbol{r}^{\prime}}\right)\right\rangle \propto \varepsilon^{\prime \prime}(\omega)$, with $\alpha, \beta=x, y, z$. In our scheme, variations of the EM field distribution and current density dispersion affect the partial and total power absorbed of the system, which are the origin of the Casimir-Lifshitz force.

\section{A. Effect on $F_{(C-L)}$ of the layer thickness and number of bilayers in multilayer structures}

Figure 2(a) shows, for the same system considered in Fig. 1(a), the reflectance spectra of a variety of multilayer 

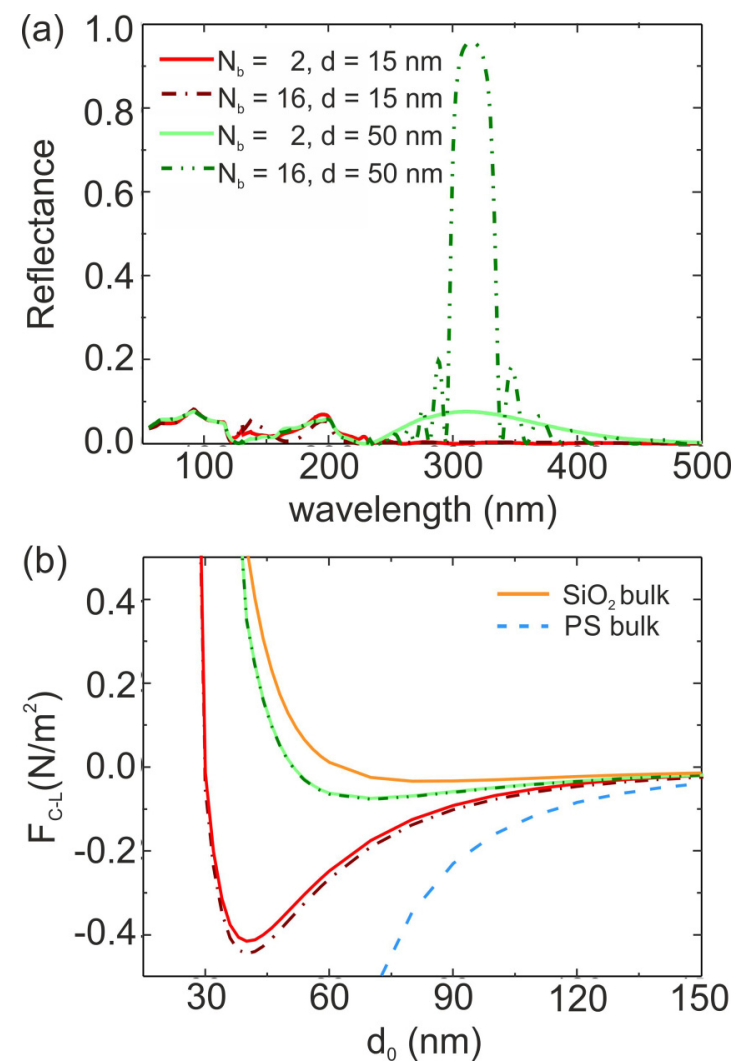

FIG. 2. (a) Reflectance spectra of a multilayer structure comprising 2 (continuous lines) or 16 (discontinuous dotted lines) bilayers of $\mathrm{SiO}_{2}$ and PS, of either $15 \mathrm{~nm}$ or $50 \mathrm{~nm}$ thickness, immersed in glycerol. The layer facing the $\mathrm{Si}$ substrate is made up of $\mathrm{SiO}_{2}$. (b) Calculation of $F_{(C-L)}$ as a function of $d_{0}$ for the systems considered in panel (a), facing a $\mathrm{Si}$ wall. As a reference, results for single slab made of either $\mathrm{SiO}_{2}$ (continuous line) or PS (dashed line) under the same conditions are also shown.

structures assuming thin layer thicknesses of $d=15 \mathrm{~nm}$ or $50 \mathrm{~nm}$, and for $N_{b}=2$ or 16 bilayers. Those thin slab dimensions result in high intensity reflectance bands emerging either at the high energetic ultraviolet range (for $d=15 \mathrm{~nm}$ ), a spectral region at which the constitutive materials strongly absorb (see Fig. 5 in Appendix A), or at near ultraviolet wavelengths (for $d=50 \mathrm{~nm}$ ), after which materials are transparent. Only for $d=50 \mathrm{~nm}$ thicknesses, typical Bragg peak characteristics can be appreciated, whereas for thinner layers of $d=15 \mathrm{~nm}$, the reflectance band at UV frequencies is greatly modulated by the strong absorption of the constitutive materials.

Figure 2(b) reveals that, for separation distances $d_{0} \leqslant$ $50 \mathrm{~nm}$, in multilayered structures the nature of the $F_{(C-L)}$ changes from being repulsive (positive) to attractive (negative) as the separation distance increases, remaining negative for large enough gaps till it vanishes. Such force behavior seems to be dictated by an interplay between the opposite force nature displayed by single slabs of $\mathrm{SiO}_{2}$ and PS, whose results are also displayed in the figure. This modulation in the force intensity is larger for thinner slabs [26] than for thicker ones, despite the largest reflectance variations being attained for thick constitutive slabs [panel (a)]. Moreover,
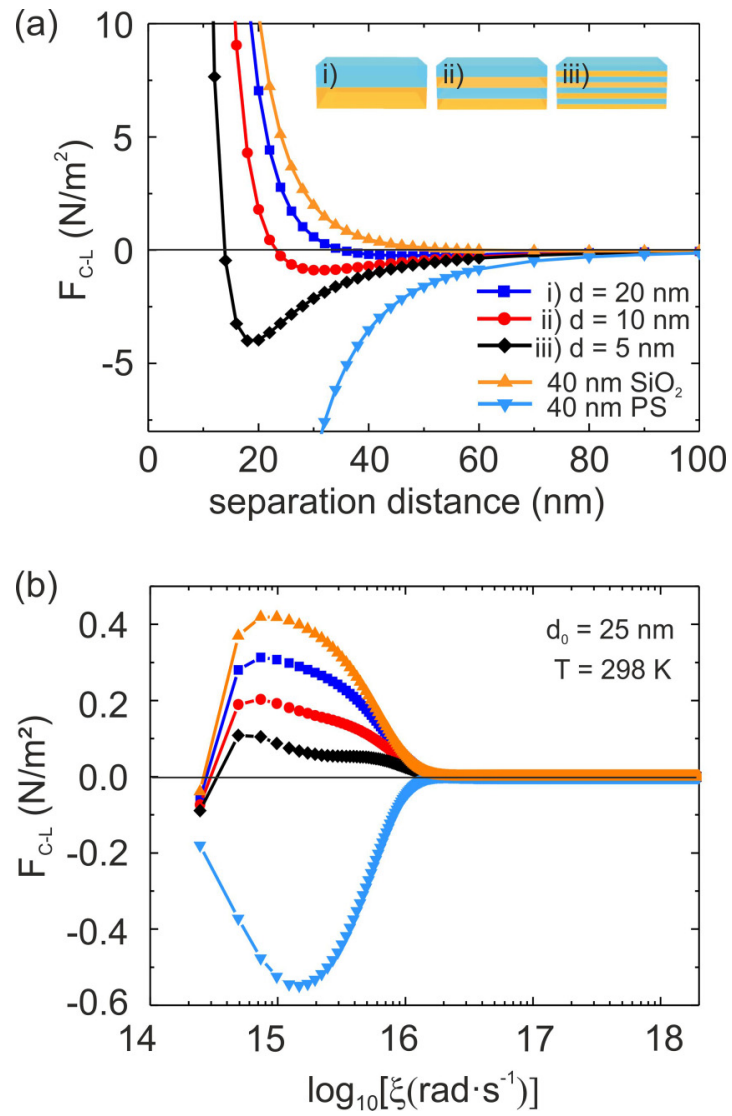

FIG. 3. (a) Calculation of $F_{(C-L)}$ as a function of $d_{0}$ for diverse multilayer structures comprising bilayers made of $50 \%$ of $\mathrm{SiO}_{2}$ and $50 \%$ of PS, with a total thickness of $40 \mathrm{~nm}$, immersed in glycerol facing a $\mathrm{Si}$ wall. Results correspond to (i) $N_{b}=1$ (i.e., $d=20 \mathrm{~nm}$ ) shown with squares, (ii) $N_{b}=2$ (i.e., $d=10 \mathrm{~nm}$ ) shown with circles, and (iii) $N_{b}=4$ (i.e., $d=5 \mathrm{~nm}$ ) shown with diamonds. As a reference, results for a $40 \mathrm{~nm}$ single slab made of either $\mathrm{SiO}_{2}$ or PS are also shown with top and down triangles, respectively. (b) For the same systems considered in panel (a), $F_{(C-L)}$ as a function of the Matsubara frequencies at a fixed separation distance $d_{0}=25 \mathrm{~nm}$. Temperature is fixed to $298 \mathrm{~K}$.

there is almost no difference between the response of 2 and 16 bilayers, demonstrating that the reaction of the multilayer system is dominated by the first bilayer, again in spite of the strong variations of the reflectance properties attained for the different number of bilayers. The former result can be explained by the fluctuation-dissipation theorem which states null current density dispersion, i.e., hence no contribution to $F_{(C-L)}$, for nonabsorbing materials $\left(\varepsilon^{\prime \prime}=0\right)$. This is the case for $\mathrm{SiO}_{2}$ and $\mathrm{PS}$ at the range of wavelengths in which the high reflectance peak appears for the multilayer with $d=50 \mathrm{~nm}$. As we will show next, our findings point to a correlation of the variations of $F_{(C-L)}$ with absorption changes due to interference effects within the nanostructured system at UV frequencies, where both $\mathrm{SiO}_{2}$ and PS bulk materials present large absorption bands. In order to gain physical insight into the last observation, systems containing the same material volumes should be compared. 
(a)



(b)

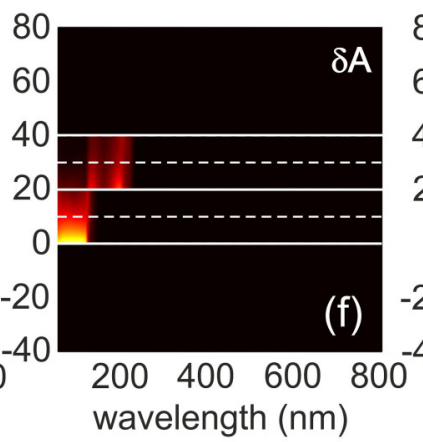

(c)

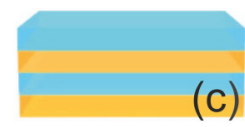

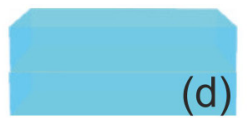

(d)
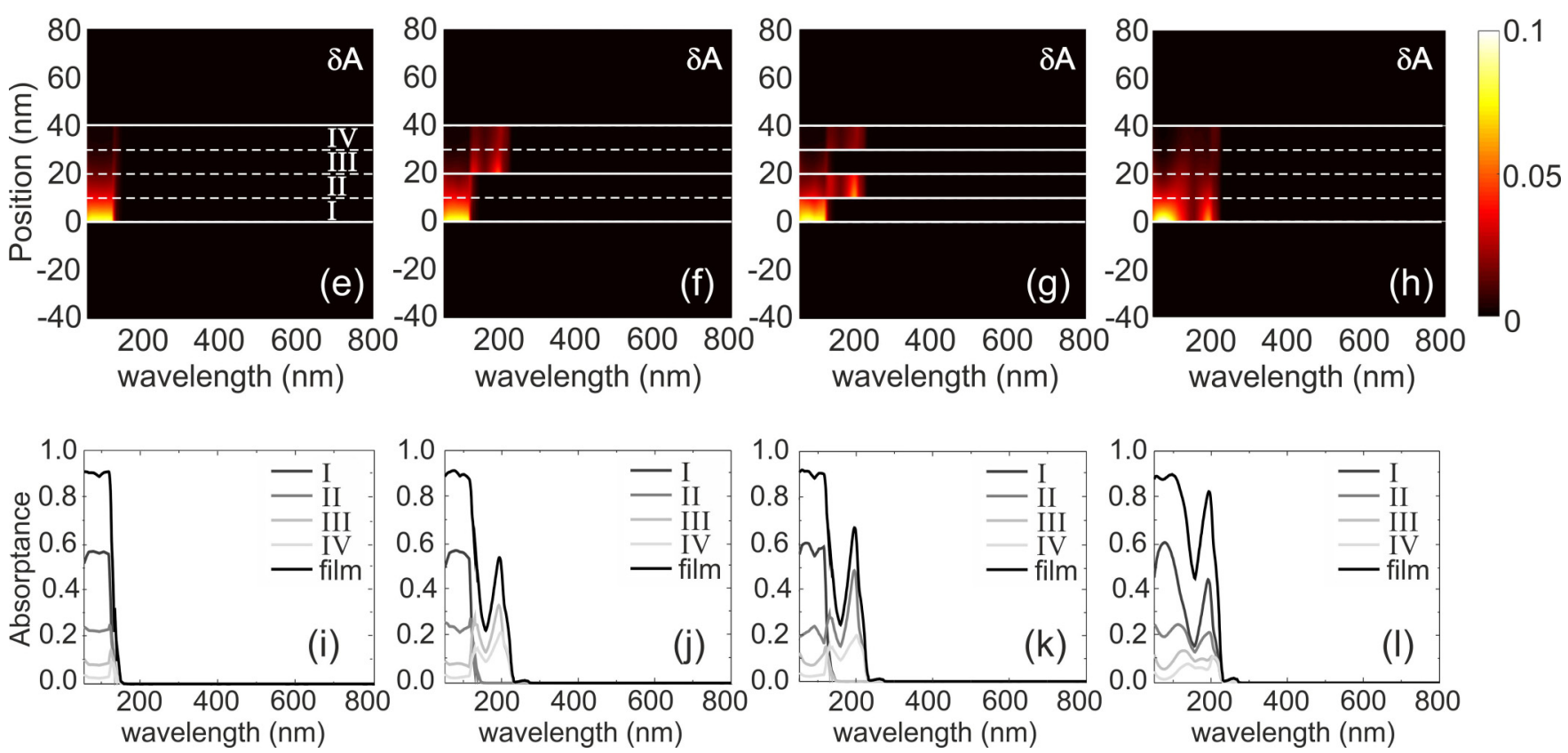

FIG. 4. Panels (a)-(d): schematics of the analyzed systems with total thickness $40 \mathrm{~nm}$, with $50 \%$ of $\mathrm{SiO}_{2}$ and $50 \%$ of PS materials. Specifically, (a) a single $\mathrm{SiO}_{2}$ slab, (b) one bilayer comprising slabs of $20 \mathrm{~nm}$ thickness, (c) two bilayers comprising slabs of $10 \mathrm{~nm}$ thickness, and (d) a single PS slab. Panels (e)-(h): corresponding spectral and spatial distribution of absorption per unit volume, $\delta A$. As a guide for the eye, limits of each material layer are marked with horizontal continuous lines and regions of equal $10 \mathrm{~nm}$ thickness with dashed ones. Panels (i)-(1): corresponding absorptance spectra displaying partial absorptance (corresponding to four consecutive regions of $10 \mathrm{~nm}$ thickness) and total absorptance (black thick line).

\section{B. Effect on $F_{(C-L)}$ of nanostructuring the same material volume in diverse multilayer configurations}

In Fig. 3(a) we consider periodic multilayer structures of $40 \mathrm{~nm}$ thickness, thin enough to yield $F_{(C-L)}$ variations by nanostructuration. Systems comprise $50 \%$ of $\mathrm{SiO}_{2}$ and $50 \%$ of PS arranged as $N_{b}=1$ (i.e., $d=20 \mathrm{~nm}$ ), $N_{b}=2$ (i.e., $d=10 \mathrm{~nm}$ ), and $N_{b}=4$ (i.e., $d=5 \mathrm{~nm}$ ) bilayers. $F_{(C-L)}$ calculations show, in all cases, repulsive forces at short separation distances $\left(d_{0} \leqslant 35 \mathrm{~nm}\right)$, which change to be attractive at larger ones, as it was already seen in Fig. 2(b). In addition, the higher the number of bilayers, which entails thinner layers, the larger the variations in $F_{(C-L)}$. Specifically, the CasimirLifshitz force can be tuned in intensity and nature through the internal nanostructure of the multilayer system while keeping the total thickness and material volumes constant. For instance, at a separation distance of $d_{0}=25 \mathrm{~nm}, F_{(C-L)}$ can be tuned from being highly repulsive with 1 bilayer, to highly attractive if it is nanostructured in 4 bilayers, even being zero if 2 bilayers are grown. This modulation of $F_{(C-L)}$ in systems in which the amount of the constituent materials is the same but their nanostructuring is different results from the interplay between attractive and repulsive forces displayed by the individual materials when considered alone. Further evidence of such interplay appears in Fig. 3(b), which shows the contribution to $F_{(C-L)}$ of each Matsubara frequency (with $n>0$ ) at $T=298 \mathrm{~K}$ and $d_{0}=25 \mathrm{~nm}$, a separation distance at which the three multilayer structures present $F_{(C-L)}$ of different nature. Specifically, the total $F_{(C-L)}$ value results from the addition of all Matsubara frequencies, i.e., those displayed in Fig. 3(b) plus the zero Matsubara frequency term. Analysis of a slab exclusively made of PS (down triangles), with $d=40$ $\mathrm{nm}$, shows that all contributions are negative (i.e., of attractive nature), including the zero Matsubara frequency term $-11.11 \mathrm{~N} / \mathrm{m}^{2}$. In this case, the addition of all Matsubara frequency contributions produces a total attractive force. In the case of a pure $\mathrm{SiO}_{2}$ material slab (up triangles), although the zero Matsubara frequency contribution is negative, -10.35 $\mathrm{N} / \mathrm{m}^{2}$, the rest of the Matsubara frequencies present positive (repulsive) contributions yielding a total force of repulsive nature. For the multilayer structures considered, in which an equal material volume is differently arranged, with (i) $d=20$ nm (squares), (ii) $d=10 \mathrm{~nm}$ (circles), and (iii) $d=5 \mathrm{~nm}$ (diamonds), the zero Matsubara frequency contribution is negative (attractive) in all cases, taking the values $-10.51 \mathrm{~N} / \mathrm{m}^{2}$, $-10.60 \mathrm{~N} / \mathrm{m}^{2}$, and $-10.68 \mathrm{~N} / \mathrm{m}^{2}$, respectively, while the contribution of all the relevant low Matsubara frequencies is always positive (at high frequencies, positive and negative contributions occur, but their impact on the final summation is negligible). Importantly, the lower the $d$ value, the lower the intensity of those contributions. Because of that, the resulting total force changes from being repulsive in structures with $d=20 \mathrm{~nm}$ to attractive in multilayers with $d=5 \mathrm{~nm}$, since 
in the latter case, the addition of all Matsubara frequencies with $n>0$ does not compensate the contribution of the zero frequency. Also, Fig. 6 in Appendix B displays reflectance and absorptance ( $A=P_{\mathrm{abs}} / P_{0}$, with $P_{0}$ the incident power) spectra of the considered arrangements.

Next, we will analyze spectral and spatial absorption profiles in such multilayers to demonstrate that, by nanostructuring the same material volume, absorption properties are modified at the UV due to optical interference, giving rise to modifications of $F_{(C-L)}$ according to the fluctuationdissipation theorem. Top panels in Fig. 4 show schematics of the systems under study, all of them with a total thickness of $40 \mathrm{~nm}$. From left to right, they correspond to (a) a single $\mathrm{SiO}_{2}$ slab of $40 \mathrm{~nm}$ thickness, (b) 1 bilayer comprising slabs of $20 \mathrm{~nm}$ thickness of $\mathrm{SiO}_{2}$ and PS layers, (c) 2 bilayers comprising $\mathrm{SiO}_{2}$ and PS slabs of $10 \mathrm{~nm}$ thickness each, and (d) a single PS slab of total thickness of $40 \mathrm{~nm}$. Much information can be attained from absorptance properties. Spectral and spatial absorption profiles along the $z$ direction are shown in the second row panels [(e)-(h)]. In them, the absorption per unit volume, $\delta A$, is depicted as being $A=\int \delta A d z$. Values of $z<0 \mathrm{~nm}$ correspond to the illumination (or reflection) region, $0 \mathrm{~nm} \leqslant z \leqslant 40 \mathrm{~nm}$ to the slab internal structure, and $z>40 \mathrm{~nm}$ to the transmission region. As a guide to the eye, limiting material interfaces are shown as continuous horizontal lines, and layers of equal $10 \mathrm{~nm}$ thickness are shown with dashed ones. Let us first analyze the absorption properties of single slabs made of $\mathrm{SiO}_{2}$ or PS (panels on the left and right columns, respectively). Notably, high absorption within the first $\approx 20 \mathrm{~nm}$ inside the $\mathrm{SiO}_{2}$ slab at $\lambda \leqslant 100 \mathrm{~nm}$ is observed, whereas PS layers absorb even deeper inside the structure (up to $\approx 40 \mathrm{~nm}$ ) and within a wider spectral range $\lambda \leqslant 200 \mathrm{~nm}$. A minimum in absorption is displayed around $\lambda \approx 150 \mathrm{~nm}$. Finally, total and partial (every $10 \mathrm{~nm}$ thickness inside the structure) absorptance spectra are shown in the fourth panels [(i)-(1)] as a volume integral of the above profiles. Corresponding electric-field distributions along the $z$ direction, $|E|^{2}$, are shown in Fig. 7 in Appendix C, along with results for 4 bilayers in Fig. 8 .

Considering now the multilayered structures, analysis of the absorption profiles [panels (f),(g)] demonstrates the existence of hot spots inside the nanostructure that develop with the number of layers, displaying regions of maximum and minimum absorption that alternate in accordance to the profiles of the single $\mathrm{SiO}_{2}$ and PS slabs previously analyzed. Furthermore, as the number of layers is increased the PS slab gets physically closer to the illumination region (because the thickness of the first $\mathrm{SiO}_{2}$ layer is reduced), having a strong impact on the partial and total spectral absorptance of the multilayer structure. To compare light absorption inside the structure, partial absorption of $10 \mathrm{~nm}$ thickness regions (denoted as I, II, III, and IV) are calculated. Since $F_{(C-L)}$ of a single PS layer is of comparable (although slightly larger) intensity and opposite nature to the force experienced by a single $\mathrm{SiO}_{2}$ layer, these results seem to indicate that $F_{(C-L)}$ is strongly modulated by the presence of the PS layer in stratified systems, and such modulation comes from absorption changes occurring at the UV range due to optical interference. Interestingly, the total spectral absorptance (black thick lines) is larger in stratified systems comprising 2 bilayers [panel (k)]
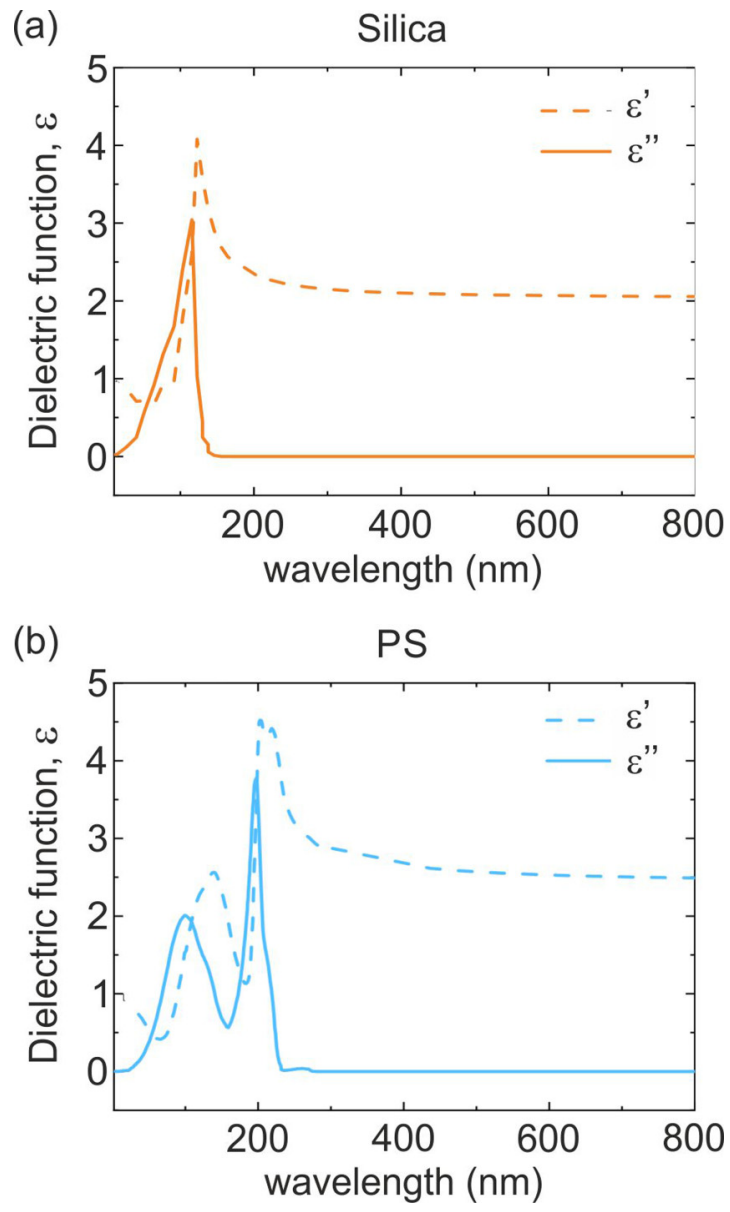

FIG. 5. Complex dielectric functions $\varepsilon$ as a function of wavelength for (a) silica and (b) polystyrene. Real $\left(\varepsilon^{\prime}\right)$ and imaginary $\left(\varepsilon^{\prime \prime}\right)$ parts of the dielectric functions are depicted with dashed and solid lines, correspondingly.

instead of only 1 bilayer [panel (j)]. The reason is that in the systems comprising 2 bilayers, more light reaches the first PS slab, since the first $\mathrm{SiO}_{2}$ layer is thinner and absorbs photons less efficiently. Our hypothesis about the different $F_{(C-L)}$

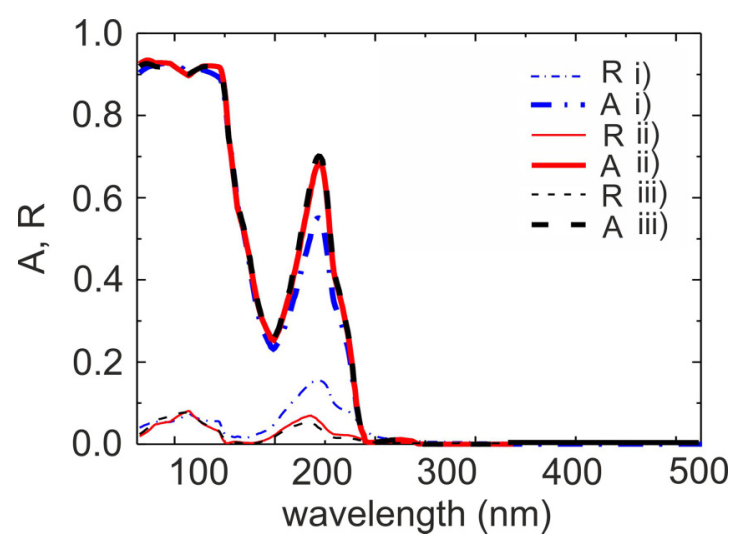

FIG. 6. Reflectance (thin solid line) and absorptance (thick solid line) for the three multilayer configurations. Results correspond to multilayers with (i) $d=20 \mathrm{~nm}$ (dotted lines), (ii) $d=10 \mathrm{~nm}$ (continuous lines), and (iii) $d=5 \mathrm{~nm}$ (dashed lines). 
(a)

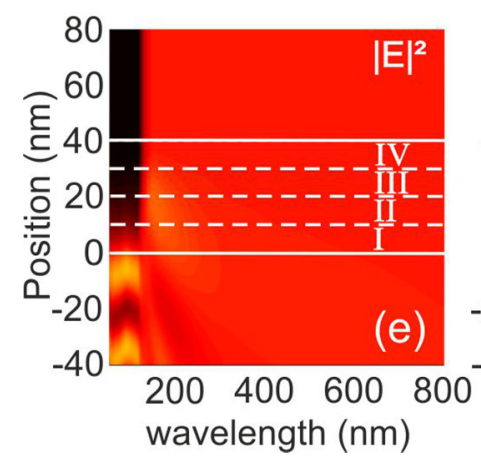

(b)

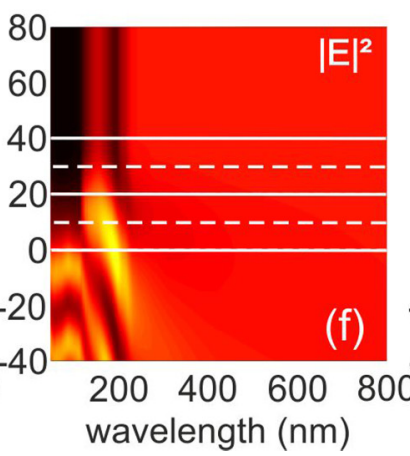





(d)





FIG. 7. Spectral and spatial distribution of $|E|^{2}$ corresponding to the same systems in Fig. 4. As a guide for the eye, limits of each material layer are marked with horizontal continuous lines and layers of equal $10 \mathrm{~nm}$ thickness with dashed ones.

experienced by the multilayer structures here considered is that, the closer the PS layer to the mediating (glycerol) layer, the stronger the variations of the absorptance properties of the stratified arrangement due to optical interference at UV wavelengths, which directly impact on $F_{(C-L)}$ according to the fluctuation-dissipation theorem. Changing the absorptance of the whole system involves modifications of the corresponding effective dielectric function. The latter translates into an adjustment of the $\varepsilon\left(i \xi_{n}\right)$ of all the interacting materials, therefore providing Casimir-Lifshitz forces of tunable intensity and nature.

\section{CONCLUSIONS}

In conclusion, we have presented a physical mechanism modifying the Casimir-Lifshitz force in dielectric multilayer nanostructures based on optical interference effects. Optical interference effects yield high electric-field localization within the nanostructure, which in turn reshapes the partial absorptance of the constituent materials at the UV frequency range, modifying the $F_{(C-L)}$ experienced by the system. Our hypothesis is based on the fluctuation-dissipation theorem that directly relates $F_{(C-L)}$ with the dissipative properties of the interacting objects, $\varepsilon_{L}^{\prime \prime}(\omega)$. Specifically, we demonstrate that, by nanostructuring the same volume of selected dielectric materials in diverse multilayer configurations experimentally accessible, forces of attractive or repulsive nature can be attained. Potential applications in the design of MEMS and NEMS systems in which dimensions and separation distances of the constituent components are fixed at the nanoscale are envisaged.

\section{ACKNOWLEDGMENTS}

We want to thank D. C. Frustaglia and C. Genet for fruitful discussions. We acknowledge support from the Spanish Ministry of Science, Innovation and Universities for funding under Grant EXPLORA No. FIS2017-91018-EXP. S.C.-P. acknowledges funding through Juan de la Cierva Incorporación under Grant No. IJCI-2016-28549. The project that gave rise to these results received the support of a fellowship granted to V.E. from la Caixa Foundation (ID No. 100010434). The fellowship code is LCF/BQ/ES15/10360025.

\section{APPENDIX A: DIELECTRIC FUNCTION OF $\mathrm{SiO}_{2}$ AND PS}

Both $\mathrm{SiO}_{2}$ and PS, the materials employed in the multilayer nanostructures here analyzed, behave as absorbing or transparent materials depending on the incident wavelength. Figure 5 displays the real (dashed line) and imaginary (solid line) parts of the dielectric functions for a wide frequency range covering the UV and visible wavelengths. Panel (a) corresponds to data of $\mathrm{SiO}_{2}$ and (b) of PS. Both materials present high absorption, i.e., high values of $\varepsilon^{\prime \prime}$, in the UV range, while $\varepsilon^{\prime \prime}$ remains zero in the visible range, meaning that the materials are transparent for visible light. In Sec. III, this optical response is related to the different contributions to $F_{(C-L)}$ through the fluctuation-dissipation theorem.

\section{APPENDIX B: EXTENDED ANALYSIS OF THE OPTICAL RESPONSE IN MULTILAYER CONFIGURATIONS}

Variations of the multilayer arrangement for a fixed material volume give rise to different $F_{(C-L)}$ values, as shown in Fig. 3, in which three different arrangements are analyzed.

Moreover, depending on the materials arrangement, the optical response varies. For the three multilayer configurations considered in Fig. 3, reflectance (thin solid line) and absorptance (thick solid line) are depicted as a function of wavelength in Fig. 6. Dotted, continuous, and dashed lines represent configurations with (i) $d=20 \mathrm{~nm}$, (ii) $d=10 \mathrm{~nm}$, and (iii) $d=5 \mathrm{~nm}$, respectively. Despite having the same amount of material in the multilayer structure (precisely, 50\% of $\mathrm{SiO}_{2}$ and $50 \%$ of PS), at specific wavelengths, arrangements with less slabs (i.e., $d=20 \mathrm{~nm}$ ) reflect light more efficiently than those more stratified, giving rise to higher absorption in such less reflective systems. 


\section{APPENDIX C: EXTENDED RESULTS OF ELECTRIC FIELD AND ABSORPTION DISTRIBUTION IN MULTILAYER CONFIGURATIONS}

The spectral and spatial distribution of the intensity of the electric field and the absorption profiles in diverse multilayer arrangements are analyzed here. Specifically, spectral and spatial electric-field intensity distribution along the $z$ direction, $|E|^{2}$, corresponding to the systems analyzed in Fig. 4, are shown in Fig. 7. Top panels display schematics of the thin films studied, all of them with total thickness of 40 $\mathrm{nm}$. From left to right, they correspond to (a) a single $\mathrm{SiO}_{2}$ slab of $40 \mathrm{~nm}$ thickness, (b) 1 bilayer comprising slabs of $20 \mathrm{~nm}$ thickness of $\mathrm{SiO}_{2}$ and PS layers, (c) 2 bilayers comprising $\mathrm{SiO}_{2}$ and PS slabs of $10 \mathrm{~nm}$ thickness each, and (d) a single PS slab. Corresponding $|E|^{2}$ profiles are shown in panels (e)-(h), in which $z<0 \mathrm{~nm}$ values correspond to the illumination (or reflection) region, $0 \mathrm{~nm} \leqslant z \leqslant 40 \mathrm{~nm}$ to the slab internal structure, and $z>40 \mathrm{~nm}$ to the transmission region. As a guide to the eye, corresponding slab thicknesses are shown as dashed white horizontal lines in the panels, whereas interfaces between two materials are represented by solid white horizontal lines. The $|E|^{2}$ distribution presents specific features for each configuration, specially in the region inside the nanostructure. At the reflection region, $|E|^{2}$ distributions show high variations at the same wavelengths at which maximum absorption of the constitutive materials occurs (see Fig. 4), and in transmission displays a local maximum at $\lambda \approx$ $150 \mathrm{~nm}$ that corresponds to a minimum of absorption in the PS spectrum.

Figure 8 gathers the information about the electric field and absorption distribution inside a multilayer nanostructure with the same material volume as those considered in Fig. 4, made up of eight alternating layers of PS and $\mathrm{SiO}_{2}$ materials. The latter entails multilayers composed by extremely thin constituent slabs of $d=5 \mathrm{~nm}$ thickness. Panel (a) corresponds to the schematic of the multilayered system, whereas (b) and (c) show the spectral and spatial distribution of the electric-field intensity and absorption inside the structure. Interestingly, hot spots develop within the multilayer structure as the PS and $\mathrm{SiO}_{2}$ materials alternate, in accordance with their high absorption wavelengths as displayed in Fig. 5. Finally, panel (d) depicts the absorptance at the first four layers of $5 \mathrm{~nm}$ (gray lines) and the total absorptance (black thick line). The
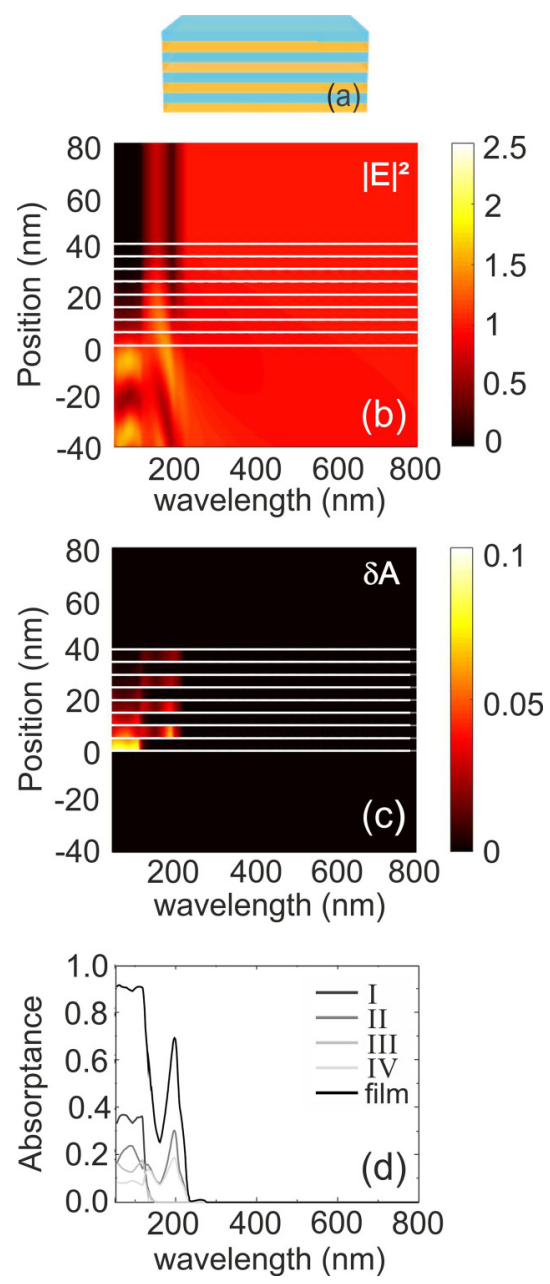

FIG. 8. (a) Scheme of a multilayer system with total thickness $40 \mathrm{~nm}$, with $50 \%$ of $\mathrm{SiO}_{2}$ and $50 \%$ of PS materials arranged in eight layers of $5 \mathrm{~nm}$ of thickness each one. (b) Spectral and spatial distribution of $|E|^{2}$. Horizontal lines mark the limits of each layer. (c) Spectral and spatial distribution of absorption per unit volume, $\delta A$. (d) Absorptance spectra for the first four layers of $5 \mathrm{~nm}$ and the total absorptance results (black thick line).

latter allows direct comparison between different multilayer arrangements.
[1] I. E. Dzyaloshinskii, E. M. Lifshitz, and L. P. Pitaevskii, The general theory of van der Waals forces, Adv. Phys. 10, 165 (1961).

[2] H. B. G. Casimir, On the attraction between two perfectly conducting plates, Proc. K. Ned. Akad. Wet. 51, 793 (1948).

[3] E. M. Lifshitz, The theory of molecular attractive forces between solids, Sov. Phys. JETP 2, 73 (1956).

[4] F. Serry, D. Walliser, and G. Maclay, The anharmonic Casimir oscillator (ACO)-The Casimir effect in a model microelectromechanical system, J. Microelectromech. Syst. 4, 193 (1996).

[5] H. B. Chan, V. Aksyuk, R. N. Kleiman, D. J. Bishop, and F. Capasso, Quantum mechanical actuation of microelectrome- chanical systems by the Casimir force, Science (NY) 291, 1941 (2001).

[6] W. Broer, G. Palasantzas, J. Knoester, and V. Svetovoy, Significance of the Casimir force and surface roughness for actuation dynamics of MEMS, Phys. Rev. B 87, 125413 (2013).

[7] M. Sedighi and G. Palasantzas, Influence of low optical frequencies on actuation dynamics of microelectromechanical systems via Casimir forces, J. Appl. Phys. 117, 144901 (2015).

[8] F. Rosa, D. A. R. Dalvit, and P. W. Milonni, Casimir interactions for anisotropic magnetodielectric metamaterials, Phys. Rev. A 78, 032117 (2008).

[9] F. Intravaia, S. Koev, I. W. Jung, A. Talin, P. Davids, R. Decca, V. Aksyuk, D. A. R. Dalvit, and D. Lopez, Strong Casimir 
force reduction through metallic surface nanostructuring, Nat. Commun. 4, 2515 (2013).

[10] A. Noto, R. Messina, B. Guizal, and M. Antezza, CasimirLifshitz force out of thermal equilibrium between dielectric gratings, Phys. Rev. A 90, 022120 (2014).

[11] D. A. T. Somers, J. L. Garrett, K. J. Palm, and J. N. Munday, Measurement of the Casimir torque, Nature (London) 564, 386 (2018).

[12] Q.-D. Jiang and F. Wilczek, Chiral Casimir forces: Repulsive, enhanced, tunable, Phys. Rev. B 99, 125403 (2019).

[13] R. Zhao, L. Li, S. Yang, W. Bao, Y. Xia, P. Ashby, Y. Wang, and X. Zhang, Stable Casimir equilibria and quantum trapping, Science 364, 984 (2019).

[14] V. Esteso, S. Carretero-Palacios, and H. Miguez, CasimirLifshitz force based optical resonators, J. Phys. Chem. Lett. 10, 5856 (2019).

[15] A. Rodriguez, A. McCauley, D. Woolf, F. Capasso, J. D. Joannopoulos, and S. G. Johnson, Nontouching Nanoparticle Diclusters Bound by Repulsive and Attractive Casimir Forces, Phys. Rev. Lett. 104, 160402 (2010).

[16] R. Esquivel-Sirvent and G. C. Schatz, Mixing rules and the Casimir force between composite systems, Phys. Rev. A 83, 042512 (2011).

[17] G. Torricelli, P. G. van Zwol, O. Shpak, G. Palasantzas, V. Svetovoy, C. Binns, B. J. Kooi, P. Joost, and M. Wuttig, Casimir force contrast between amorphous and crystalline phases of aist, Adv. Funct. Mater. 22, 3729 (2012).

[18] V. Esteso, S. Carretero-Palacios, and H. Miguez, Nanolevitation phenomena in real plane-parallel systems due to the balance between Casimir and gravity forces, J. Phys. Chem. C 119, 5663 (2015).

[19] E. Y. Santiago, J. E. Prez-Rodrguez, and R. Esquivel-Sirvent, Dispersive properties of mesoporous gold: van der Waals and near-field radiative heat interactions, J. Phys. Chem. C 121, 12392 (2017).

[20] Y. Ye, Q. Hu, Q. Zhao, and Y. Meng, Casimir repulsiveattractive transition between liquid-separated dielectric metamaterial and metal, Phys. Rev. B 98, 035410 (2018).

[21] A. Kravtsov Yurii, I. Tatarskii Valeryan, and S. M. Rytov, Principles of Statistical Radiophysics 3. Elements of Random Fields (Springer-Verlag, Berlin, 1989).

[22] L. H. Ford, Spectrum of the Casimir effect and the Lifshitz theory, Phys. Rev. A 48, 2962 (1993).

[23] R. Esquivel-Sirvent, C. Villarreal, and G. Cocoletzi, Superlattice-mediated tuning of Casimir forces, Phys. Rev. A 64, 052108 (2001).

[24] F. Pinto, Computational considerations in the calculation of the Casimir force between multilayered systems, Int. J. Mod. Phys. A 19, 4069 (2004).

[25] C. Henkel and K. Joulain, Casimir force between designed materials: What is possible and what not, Europhys. Lett. 72, 929 (2007).

[26] N. Inui, Interaction force between ultrathin multilayer films induced by quantum fluctuations, Thin Solid Films 631, 132 (2017).

[27] M. Bordag, G. L. Klimchitskaya, U. Mohideen, and V. M. Mostepanenko, Advances in the Casimir Effect (Oxford Science Publications, Oxford, 2009).
[28] M.-S. Tomas, Green function for multilayers: Light scattering in planar cavities, Phys. Rev. A 51, 2545 (1995).

[29] F. Zhou and L. Spruch, van der Waals and retardation (Casimir) interactions of an electron or an atom with multilayered walls, Phys. Rev. A 52, 297 (1995).

[30] C. Genet, A. Lambrecht, and S. Reynaud, The Casimir force and the quantum theory of lossy optical cavities, Phys. Rev. A 67, 043811 (2002).

[31] M.-S. Tomas, Casimir force in absorbing multilayers, Phys. Rev. A 66, 052103 (2002).

[32] C. Raabe, L. Knöll, and D.-G. Welsch, Three-dimensional Casimir force between absorbing multilayer dielectrics, Phys. Rev. A 68, 033810 (2002).

[33] R. Podgornik, P. Hansen, and V. Parsegian, On a reformulation of the theory of Lifshitz-van der Waals interactions in multilayered systems, J. Chem. Phys. 119, 1070 (2003).

[34] R. Podgornik, R. French, and V. Parsegian, Nonadditivity in van der Waals interactions within multilayers, J. Chem. Phys. 124, 044709 (2006).

[35] B. E. Sernelius, Electromagnetic normal modes and Casimir effects in layered structures, Phys. Rev. B 90, 155457 (2014).

[36] V. Esteso, S. Carretero-Palacios, and H. Miguez, Effect of temperature variations on equilibrium distances in levitating parallel dielectric plates interacting through Casimir forces, J. Appl. Phys. 119, 144301 (2016).

[37] R. Kitamura, L. Pilon, and Miroslaw Jonasz, Optical constants of silica glass from extreme ultraviolet to far infrared at near room temperature, Appl. Opt. 46, 8118 (2007).

[38] U. Strom, J. R. Hendrickson, R. J. Wagner, and P. C. Taylor, Disorder-induced far infrared absorption in amorphous materials, Solid State Commun. 15, 1871 (1974).

[39] T. Inagaki, E. T. Arakawa, R. N. Hamm, and M. W. Williams, Optical properties of polystyrene from the near infrared to the $\mathrm{X}$-ray region and convergence of optical sum rules, Phys. Rev. B 15, 3243 (1977).

[40] W. R. Folks, S. K. Pandey, G. Pribil, W. D. Slafer, M. Manning, and G. Boreman, Reflective infrared ellipsometry of plastic films, Int. J. Infrared Millimeter Waves 27, 1553 (2006).

[41] L. Duraffourg and P. Andreucci, Casimir force between doped silicon slabs, Phys. Lett. A 359, 406 (2006).

[42] D. F. Edwards, Handbook of Optical Constants of Solids, 1st ed. (Academic Press, New York, 1998).

[43] P. J. van Zwol and G. Palasantzas, Repulsive Casimir forces between solid materials with high-refractive-index intervening liquids, Phys. Rev. A 81, 062502 (2010).

[44] P. Yeh, Optical Waves in Layered Media (John Wiley \& Sons, New York, 1988).

[45] E. Hecht, Optics (Addison-Wesley, Redwood City, CA, 2002).

[46] R. D. Birkhoff, L. R. Painter, and J. M. Heller, Optical and dielectric functions of liquid glycerol from gas photoionization measurements, J. Chem. Phys. 69, 4185 (1978).

[47] J. Rheims, J. Köser, and T. Wriedt, Refractive-index measurements in the near-IR using an abbe refractometer, Meas. Sci. Technol. 8, 601 (1997). 